\title{
REFLECTED FORWARD BACKWARD STOCHASTIC DIFFERENTIAL EQUATIONS AND CONTINGENT CLAIMS
}

Michael Kohlmann

Universitaet Konstanz, Germany

\section{INTRODUCTION}

In [8] we examined the properties of adjoint processes in stochastic control on the basis of the theory of stochastic flows. We extended earlier results by [2] and made extensive use of the important contributions to the theory by Pardoux, Peng, Zhou, and Renner, and of the results on stochastic partial differential equations by Antonelli, Pardoux, Peng, Zhou, which extend the pioneering works by Bensoussan, Bismut, Kunita, and many others.For references the reader is referred to [8]. We will here apply some of the results to the theory of pricing contingent claims. The valuation process will take the form of a possibly reflected BSDE (see e.g.[3]) which will be interpreted as the adjoint of a trivial, possibly singular control problem. Using the results in [1] we study sequential hedging problems.

\section{A TRIVIAL CONTROL PROBLEM}

A bond/stock price-asset is given in the usual notation by

$$
\begin{gathered}
d P_{t}^{0}=r\left(P_{t}^{0}, \omega\right) d t \text { (bond) } \\
d P_{t}^{i}=b_{i}\left(t, P_{t}, \omega\right) d t+\sigma_{i j}\left(t, P_{t}, \omega\right) d w_{t}^{j} \text { (stock) }
\end{gathered}
$$

on the time interval $[0, T]$. Let $\left(P_{t}^{s x}\right)$ be the strong solution of the SDE with initials $(s, x) \in[0, T] \times \Re^{n}$, and let $\sigma$ satisfy conditions such that a unique risk premium process

$$
\theta\left(t, P_{t}, \omega\right)=\sigma^{-1}\left(t, P_{t}, \omega\right)\left[b\left(t, P_{t}, \omega\right)-r\left(t, P_{t}, \omega\right) \cdot 1_{n}\right]
$$

exists. With this we are in an arbitrage free world (for details see e.g. [7]).We will assume that $n=d=1$. In the first part of this article this is no real restriction. Most results can be generalized to higher dimensions. In the last section, however, this assumption becomes crucial, as there we make use of

The original version of this chapter was revised: The copyright line was incorrect. This has been corrected. The Erratum to this chapter is available at DOI: 10.1007/978-0-387-35359-3_40 
comparison theorems where processes are used which have similar properties as local times. As in [1] this makes an extension to higher dimensions impossible, at least at the moment. Also we should note that some of the results on spdes used below only hold when $\left(P_{t}\right)$ is replaced by $\left(\log P_{t}\right)$.

Now consider the following trivial control problem

$$
\begin{gathered}
d z_{s t}=-z_{s t}\left[r\left(t, P_{t}^{s x}, \omega\right) d t+\theta\left(t, P_{t}^{s x}, \omega\right) d w_{t}\right] \\
z_{s s}=1
\end{gathered}
$$

with cost criterion $J=E\left[z_{s T} g\left(P_{T}^{s x}\right)\right]$, where $g: \Re \times \Omega \rightarrow \Re^{+}$is a nonnegative, bounded, non-anticipative process which is assumed to be once continuously differentiable in the first variable. We interpret this as a control problem with a trivial one-point control space. The formal adjoint process for this control problem is given by the backward equation ( more exactly we had to call it a system of forward-backward sdes)

$$
y_{t}=g\left(P_{T}^{s x}\right)-\int_{t}^{T}\left[y_{u} r\left(u, P_{u}^{s x}\right)+Z_{u} \theta\left(u, P_{u}^{s x}, \omega\right)\right] d u-\int_{t}^{T} Z_{u} d w, t \in[s, T] .
$$

Note that $\left(z_{s t}\right)$ is the deflator process and $\left(y_{t}\right)$ is the price process for the claim $\xi_{T}:=g\left(P_{T}^{s x}\right)$, where the formal duality gives the interpretation

$$
y_{t}=E\left[z_{t T} \xi_{T} \mid F_{t}\right], t \in[s, T]
$$

Rewrite $\left(y_{t}\right)$ as $y_{t}=\bar{E}\left[\exp \left(-\int_{t}^{T} r\left(s, P_{s}\right) d s\right) \xi_{T} \mid F_{t}\right]$, where $\bar{E}$ is the expectation with respect to the risk neutral measure associated with the Girsanov functional of $\theta$. In this form we see that $\left(y_{t}\right)$ corresponds to the risk neutral price of the claim in the classical notation. Also note that $z_{s t} y_{t}$ is a $\mathrm{P}$-martingale. So, in order to determine the price of the claim it is necessary to solve the BSDE.

Definition A solution of the BSDE

$$
y_{t}=g\left(P_{T}^{s x}\right)-\int_{t}^{T}\left[y_{u} r\left(u, P_{u}^{s x}\right)+Z_{u} \theta\left(u, P_{u}^{s x}, \omega\right)\right] d u-\int_{t}^{T} Z_{u} d w_{u}
$$

is a pair $(y, Z)$ such that $\left(y_{t}\right)$ is a continuous, adapted process and $\left(Z_{t}\right)$ is a predictable, square integrable process. The solution is unique if both processes are equal $P-a . s$.. [3].

Conditions to guarantee a unique solution in the sense of 2.1 are found in

Now we will try to characterize the solution in terms of a pricing system. This is defined to be a mechanism to bring $\left(y_{t}\right)$ and $\left(P_{t}\right)$ into a relation.

\section{THE PRICING SYSTEM}

Definition A stochastic pricing system for the claim $\xi_{T}$ is a function $u:[0, T] \times$ $\Re \times \Omega \rightarrow \Re$ which satisfies $u$ is progressively measurable and $u\left(t, P_{t}^{s x}, \omega\right)=y_{t}(\omega)$ $P-a . s ., t \in[s, T]$.

At this stage we do not impose conditions in the second variable, as such conditions must comply with the real world requirements. 
Definition The pricing system will be called convenient, if $u(t, ., \omega)=$ $u\left(t, ., \alpha_{t}\right)$

where $\alpha$ is a given diffusion process. It will be called differentiable, if $u(t, ., \omega) \in C^{12} P-a . s$, and it will be called deterministic if $u(t, x, \omega)=u(t, x)$.

a) the most general case

Let $E\left[z_{t T} g\left(P_{T}^{t x}\right) \mid F_{t}\right]=u(t, x, \omega)$, where $x=P_{t}^{s x}$. As we may assume that $E\left[z_{t T} g\left(P_{T}^{t x}\right) \mid F_{t}\right]$ is a special semimartingale (under appropriate conditions) we write $u(t, x, \omega)$ as an integral equation between random fields (see [9] for semimartingales with spatial parameters):

$$
u(t, x, \omega)=u(0, x, \omega)+\int_{0}^{t} p(s, x, \omega) d s+\int_{0}^{t} k(s, x, \omega) d w_{s} .
$$

Now apply the Itô-Ventcell formula as generalized in [9] to find

$$
\begin{aligned}
u\left(t, P_{t}\right)= & u\left(T, P_{T}\right)-\int_{t}^{T}\left[p\left(s, P_{s}\right)+1 / 2 \sigma^{2}\left(s, P_{s}\right) u_{x x}\left(s, P_{s}\right)\right] d s \\
& -\int_{t}^{T}\left[b\left(s, P_{s}\right) u_{x}\left(s, P_{s}+\sigma\left(s, P_{s}\right) k_{x}\left(s, P_{s}\right)\right]\right. \\
& -\int_{t}^{T}\left[k\left(s, P_{s}\right)+\sigma\left(s, P_{s}\right) u_{x}\left(s, P_{s}\right)\right] d w_{s}
\end{aligned}
$$

Compare this to the backward s.d.e for $\left(y_{t}\right)$

$$
y_{t}=g\left(P_{T}^{s x}\right)-\int_{t}^{T}\left[y_{u} r\left(u, P_{u}^{s x}\right)+Z_{u} \theta\left(u, P_{u}^{s x}, \omega\right)\right] d u-Z_{u} d w_{u}
$$

to find

$$
\begin{aligned}
u\left(T, P_{T}\right) & =g\left(P_{T}\right)=\xi_{T} \\
p & =-1 / 2 \sigma^{2} u_{x x}-b u_{x}-\sigma k_{x}+r u+\left(k+\sigma u_{x}\right) \theta \\
& =-1 / 2 \sigma^{2} u_{x x}-(b-\sigma \theta) u_{x}+r u+k \theta-\sigma k_{x}
\end{aligned}
$$

where $Z=k+\sigma u_{x}$.This means that the solution of the spde

$$
u_{t}=\left[1 / 2 \sigma^{2} u_{x x}+(b-\sigma \theta) u_{x}-r u-k \theta+\sigma k_{x}\right] d s-k d w
$$

with final condition $u(T, x)=g(x)$ is a stochastic price system in the sense of definition 3.1.

Remark (i) For conditions to ensure existence and uniqueness of this spde the reader is referred to [10].

(ii) When we take the hedging point of view as in [7] it is staightforward that the optimal hedging strategy is given by $\pi_{t}=\sigma^{-1} Z_{t}=u_{x}\left(t, P_{t}\right)+$ $\sigma^{-1}\left(t, P_{t}\right) k\left(t, P_{t}\right)$. 
(iii) Many arguments above become notationally more transparent when we use notations and results from Malliavin's calculus. However, in order to make the results easily comparable to the application below, we refrained from doing so.

(iv) The result $k=Z-\sigma u_{x}$ is closely related to an equation which appears in the maximum principle of a stochastic control problem, where both drift and diffusion are controlled. There the term corresponds to the second adjoint equation. An important question then arises, namely when equality holds between $Z$ and $\sigma u_{x}$, i.e. $k_{t}=0$.

(v) The BSDE as a tool to model evaluations of claims has turned out to be extremely powerful. So it is easy to model the Foellmer-Schweizer hedging within this model: just subtract a martingale orthogonal to the Brownian motion from the original BSDE:

$$
y_{t}=g\left(P_{T}^{s x}\right)-\int_{t}^{T}\left[y_{u} r\left(u, P_{u}^{s x}\right)+Z_{u} \theta\left(u, P_{u}^{s x}, \omega\right)\right] d u-Z_{u} d w_{u}-M_{t}
$$

Or just as another example for the power of this tool: Recently we could derive the price and portfolio of an informed agent, i.e. an agent with anticipative knowledge about part of the market, by applying the BSDE-techniques to Protter's [11]result on the connection between the enlargement of a filtration and Girsanov's theorem. This simplifies the proof [6], and extends the result to include the Foellmer-Schweizer model.

The BSDE technique appears to be tailor made for finance purposes.

To compute the price of the claim and the optimal hedging strategy we have to

(i) solve the FBSDE and compute $\sigma^{-1} Z_{t}$ or equivalently

(ii) solve the spde $(u, k)$ and compute $u_{x}\left(t, P_{t}\right)+\sigma^{-1} k\left(t, P_{t}\right)$

$$
\text { or }
$$

(iii) find cases where $k=0$ and then compute $u_{x}\left(t, P_{t}\right)$ with different means.

b) the convenience rate case

To solve the problem of 3.3 (iii) we use a result in [12] where the relation between first and second adjoint was considered in the framework of stochastic control. From the point of view of finance our setting will be more general than necessary for the convenience rate problem, where only the drift coefficient would depend on a further forward SDE. However we hope to treat more general problems as e.g. a problem of pricing an asset which depends on an index of some kind in the coefficients:

Let the index be described by

$$
d \alpha_{t}=a\left(t, \alpha_{t}\right) d t+c\left(t, \alpha_{t}\right) d w_{t}, a_{0}=\alpha
$$

and let all randomness in $g, r, b, c$ come from $\left(P_{t}, \alpha_{t}\right)$, i.e. 


$$
d P_{t}=b\left(t, P_{t}, \alpha_{t}\right) d t+\sigma\left(t, P_{t}, \alpha_{t}\right) d w_{t}
$$

In this case a quite lengthy technical computation leads to the result

$$
k_{s}=Z_{s}-\sigma u_{x}-\sigma u_{\alpha}=0
$$

so that here we find a deterministic price system in the form $u(t, x, \alpha)$. The influence of the index on the portfolio is similar to the influence of the volatility of the stock price.

\section{c) direct computations}

Finally let us apply some results from the theory of stochastic flows to generate an explicit representation of $\left(y_{t}\right)$. We here assume that we are in a Markovian world, that all coefficients are sufficiently differentiable, and that $r=0$. The last assumption is made at the beginning to make the results from [2] applicable without change. The general case then is a simple obvious generalization. We are now going to compute the representation of the martingale

$$
y_{t}=\bar{E}\left[g\left(P_{T}\left(x_{0}\right)\right) \mid F_{t}\right]=\bar{E}\left(g\left(P_{T}\left(x_{0}\right)\right)\right)+\int_{0}^{t} \gamma d \widetilde{w}
$$

where $\widetilde{w}$ is a Brownian motion under the risk neutral measure $\widetilde{P}$. From the Markov property we have

$$
\begin{aligned}
y_{t} & =\bar{E}\left[g\left(P_{T}\left(x_{0}\right)\right) \mid F_{t}\right]=E\left[z_{t T}(x) g\left(P_{t T}(x)\right) \mid F_{t}\right] \\
& =E\left[z_{t T}(x) g\left(P_{t T}(x)\right)\right]=u(t, x) .
\end{aligned}
$$

Arguing as in [4] the integrand $\left(\gamma_{s}\right)$ must be equal to

$$
\frac{\partial u}{\partial x}\left(s, P_{0 s}\left(x_{0}\right)\right) \sigma\left(s, P_{0 s}\left(x_{0}\right)\right) .
$$

It is immediate from [4] that then for the general case $r=r\left(t, P_{t}\right) \not 0$

$$
\begin{aligned}
\frac{\partial u}{\partial x}= & \\
& E\left[z _ { t T } ( x ) \left\{\int_{t}^{T}\left(\theta_{\xi}\left(r, P_{t r}\right) D_{t r} d \tilde{w} r+\int_{t}^{T} r_{\xi}\left(r, P_{t r}\right) D_{t r} d r\right) \cdot g\left(P_{0 T}\left(x_{0}\right)\right)\right.\right. \\
& \left.\left.+g_{\xi}\left(P_{t T}(x)\right) D_{t T}(x)\right\}\right],
\end{aligned}
$$

and with this we are back in the classical case.

Remark (i) As in [4] we can derive a bpde for $\gamma$.

(ii) By repeating the representation in c) over and over we find a chaos decomposition as in [5]. From this we can compute the ratios of the claim. 


\section{THE AMERICAN CONTINGENT CLAIM}

An American option allows to choose the exercise time at any time within the horizon. In order to hedge the risk of early exercise, so-called super-strategies have to be introduced. The price of the option then takes the form of a reflected backward sde as studied in [3].

Definition The reflected backward sde is described by

$$
y_{t}=\xi_{T}-\int_{t}^{T}\left[r y_{s}+\theta Z_{s}\right] d s-\int_{t}^{T} Z_{s} d w_{s}+\int_{t}^{T} d C_{t}
$$

subject to the constraints (i) $y_{t} \geq \xi_{t}$ and (ii) $\int_{0}^{T}\left(y_{t}-\xi_{t}\right) d C_{t}=0$.

A solution is a triple $\left(y^{s x C}, Z^{s x C}, C^{s x}\right)$ where $(y, Z)$ has the usual properties and $C$ is an adapted, continuously increasing process with $\mathrm{C}_{0}^{s x}=0$, such that (i) and (ii) holds for $\xi_{t}=g\left(P_{t}^{s x}\right)$.

The coefficients here have the same general properties as in the first section. The price $\left(y_{t}\right)$ is then given by

$$
y_{t}=e s s-\sup _{\tau \in[t, T]} E\left[z_{t \tau} \xi_{\tau} \mid F_{t}\right], \tau \text { stopping time. (*) }
$$

This is obvious from the following control-theoretical considerations:

$\left(y_{t}\right)$ is the first adjoint of a singular control problem. On the other hand this first derivative coincides with the value function of a stopping problem, and this is the intuitive meaning of $(*)$.

In [1] we extended the above mentioned results to general diffusions and established the relation between singular control and optimal stopping for this generalized case. Then it was shown that the singularly influenced process corresponds to a process conctructed from a monotone sequence of stopping times.

Before we go into this problem we state the following variational result for the stochastic pricing system of the American claim.

Theorem 1 : Let $u(t, x)$ be a random field which solves the obstacle problem

$$
\begin{gathered}
\{(u(t, x)-g(x)) \wedge \\
\left.\left(u(t, x)-g(x)+\int_{t}^{T} \frac{1}{2} \sigma^{2} u_{x x}+r u_{x}-r u+\sigma k_{x}-k \theta d s+\int_{t}^{T} k d w_{s}\right)\right\} \\
=0
\end{gathered}
$$

with final condition $u(T, x)=g(x)$.

This system of variational equalities (in an appropriate space) gives the stochastic price of the American contingent claim $u\left(t, P_{t}\right)=y_{t}$.

From this it is clear that the role of the increasing process $\left(C_{t}\right)$ is to keep $\left(y_{t}\right)$ away from the obstacle (or the forbidden region) $\xi_{t}$ ( $y_{t}<\xi_{t}$, respectively). This will allow the seller of the option to fulfill the requirements of the option at any time in $[0, T]$. Now it is easy to guess, a bit more difficult to prove, but standard, that the optimal stopping time is given by

$$
\tau_{t}=\inf \left\{T \geqslant s \geq t: y_{s}=\xi_{s}\right\}=\inf \left\{T \geq s \geq t: y_{s}=g\left(P_{s}\right)\right\} .
$$

Thus $\tau_{t}$ is the first time of the first move of $\left(C_{t}\right)$, and it is immediate that the American price is the European price with (random) exercise time $\tau_{t}$. 
In [1] we considered the mathematics behind a problem of installment options and related this to a problem of sequential stopping which turned out to be the limit of a family of impulse control problems. We will apply these results to describe sequential hedging. The idea is easily described: We consider the price of an American claim as decribed above by a RBSDE. At the first exercise time $\tau_{t}$ the seller offers a new option starting in $\left(\tau_{t}, P_{\tau_{t}}\right)$ and we compute the price of this new claim with these new initials, to get a second stopping time

$$
\tau_{\tau_{t}}=\inf \left\{T \geq s>\tau_{t}: y_{s}^{\tau_{t} \xi_{\tau_{t}}}=g\left(P_{s}^{\tau_{t} P_{r_{t}}}\right)\right\}
$$

The price again coincides with the European price with starting parameters $\left(\tau_{t}, P_{\tau_{t}}\right)$ and exercise time $\tau_{\tau_{t}}$. In this way we get an increasing sequence of stopping times. On the other hand let us work backwards to consider an increasing sequence of stopping times $\left(\tau_{j}\right)_{j=1, \ldots, n+1}$ and a related obviously increasing family of deterministic states $0=x_{1} \leq x_{2} \leq \ldots \leq x_{n} \leq K$ such that

$$
y_{t}^{s x\left(\tau_{j} x_{j}\right)}=g\left(P_{T}^{s x}\right)-\int_{t}^{T}\left(r\left(P_{u}^{s x}\right) y_{u}+\theta\left(P_{u}^{s x}\right) Z_{u}\right) d u-\int_{t}^{T} Z_{s} d w_{s}+x_{n}-x_{j}
$$

for $\tau_{j} \leq t<\tau_{j+1}$, where the $x_{j}$ are minimal such that $y_{t}^{s x\left(\tau_{j} x_{j}\right)} \geq \xi_{t}$ for $\tau_{j} \leq t<\tau_{j+1}$. Define $\left(\zeta_{t}^{n}\right)$ by $\zeta_{t}^{n}=x_{j}$ for $\tau_{j} \leq t<\tau_{j+1}$, so that $\left(\zeta_{t}^{n}\right)$ is increasing and right continuous. The resulting impulsed process may thus be identified with a process which at random times $\tau_{j+1}$ jumps to a process with final condition $g\left(P_{t}^{s x}\right)+\left(x_{n}-x_{j}\right)$ and stays there until $\tau_{j}$.

By choosing more and more support points it was then proved that finally there is a one-to-one correspondence between an exhaustive family of stopping times $\tau^{*}=\left(\tau_{y}\right)_{y \in J}$ derived from $\tau_{j}=\tau_{x_{j}}$ and an increasing continuous process $\left(\zeta_{t}\right)$ defined as the limit of the $\left(\zeta_{t}^{n}\right)$ constructed from $\left(\tau_{j}\right)$. $\left(\zeta_{t}\right)$ is independent of the approximating sequences so that we identify $\left(y^{s x \zeta}, Z^{s x \zeta}, \zeta\right)=$ $\left(y^{s x \tau^{*}}, Z^{s x \tau^{*}}\right)$.

Now let $\left(y^{s x C}, Z^{s x C}, C^{s x}\right)$ be a solution of the RBSDE above, then $\left(y^{s x C}, Z^{s x C}, C^{s x}\right)=\left(y^{s x \tau^{*}}, Z^{s x \tau^{*}}\right)$, so that we may summarize:

Theorem 2 A self-financing superprice of the American claim is a solution of the $R B S D E$

$$
y_{t}=\xi_{T}-\int_{t}^{T}\left[r y_{s}+\theta Z_{s}\right] d s-\int_{t}^{T} Z_{s} d w_{s}+\int_{t}^{T} d C_{t}
$$

with obstacle $y_{t} \geq \xi_{t}=g\left(P_{t}^{s x}\right)$ such that $C_{0}=0$ and $\int_{0}^{T}\left(y_{u}-\xi_{u}\right) d C_{u}=0$.

The solution is denoted by $\left(y^{s x C}, Z^{s x C}, C^{s x}\right)$. Let the correspondence between $C$ and $\tau^{*}$ be denoted by $\alpha(C)=\tau^{*}, \pi\left(\tau^{*}\right)=C$, then $\left(y^{s x \pi\left(\tau^{*}\right)}, Z^{s x \pi\left(\tau^{*}\right)}, \pi\left(\tau^{*}\right)\right)=$ $\left(y^{s x \tau^{*}}, Z^{s x \tau^{*}}\right)$ is a self-financing superprice.

For obvious reasons we call this price the price corresponding to the rolling hedge $\left(\sigma^{-1} Z^{s x \alpha(C)}\right)$. In this way the superprice is characterized by the limit of European prices with random exercise times. 


\section{References}

[1] Boetius, F.; Kohlmann, M.: Connections between optimal stopping and singular stochastic control; to appear in Stochastic Proc. Appl. (1998)

[2] Baras, J.M.; Elliott, R.J.; Kohlmann, M.: The partially observed stochastic minimum principle; SIAM Journal Control Optim. 27,6 (1989), 1279-1292

[3] El Karoui, N.; Peng, S.; Quenez, M.C.: Backward stochastic differential equations in finance; to appear in: Mathematical Finance

[4] Elliott, R.J.; Kohlmann, M.: The second order minimum principle and adjoint processes; Stochastics and Stochastics Rep. 46 (1994), 25-39

[5] Elliott, R.J.; Kohlmann, M.: Integration by parts, homogeneous chaos expansions and smooth densities; Annals of Prob. 17 (1989), 194-207

[6] Elliott,R.J.; Geman, H.; Korkie, B.M.: Portfolio optimization and contingent claim pricing with differential information; Sochastics and Stochastics Rep.60 (1997); 185-203

[7] Karatzas, I.; Shreve, S.E.: Methods of Mathematical Finance, to appear (1998)

[8] Kohlmann, M.: Adjoint equations in stochastic control, working paper (1998)

[9] Kunita, H.: Stochastic flows and stochastic differential equations, Cambridge University Press (1990)

[10] Ma, Y.; Yong, J.: Adapted solutions of a degenerate backward spde withapplications; Stochastic Proc. App. 70 (1997), 59-84

[11] Protter, P.: A connection between the expansion of filtrations and Girsanov's Theorem;in Stoch.Partial Diff. Eq. Appl. II, ed. Da Prato, G. Tubaro, L.; LNMath 1390, Springer Verlag, Berlin-Heidelberg-New York (1990)

[12] Renner, P.; Optimal control of diffusions, thesis Konstanz (1996) 